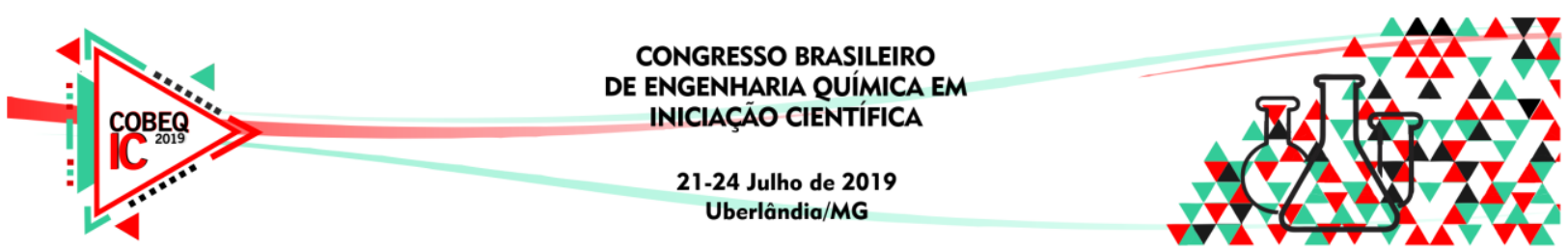

\title{
ESTUDO DAS CONDIÇÕES DE SÍNTESE DA CELULOSE MICROCRISTALINA OBTIDA A PARTIR DE CELULOSE BRANQUEADA DE BAGAÇO DE CANA
}

\author{
G. O. L. SANTOS ${ }^{1}$, S. A. Y. KAWASHIMA ${ }^{1}$, E.J.C MORAES ${ }^{1}$ e L. F. F. FARIA ${ }^{1}$ \\ ${ }^{1}$ Universidade de São Paulo, Escola de Engenharia Química \\ E-mail para contato: figue@usp.br
}

\begin{abstract}
RESUMO - Este trabalho tem como objetivo a obtenção de celulose microcristalina (MCC) utilizando o método da hidrólise ácida visando à otimização de algumas condições da reação, como a concentração de ácido, razão de massa de celulose por volume de ácido, tempo de reação e temperatura. A matéria prima utilizada foi o bagaço de cana, submetido a vários tratamentos químicos visando à separação das principais frações, obtendo assim uma polpa celulósica mais purificada apta a ser hidrolisada. Para isso foi proposto um planejamento experimental exploratório do tipo fatorial fracionado, a fim de minimizar esforço experimental com economia de tempo e recursos. Como resultado, foi observado que a condição experimental de obtenção de MCC que apresentou os melhores resultados foi concentração de $1,5 \mathrm{~mol} / \mathrm{L}$ de $\mathrm{H}_{2} \mathrm{SO}_{4}$, razão massa/solução de $1: 15(\mathrm{~g} / \mathrm{mL})$, temperatura de $85^{\circ} \mathrm{C}$ e tempo reacional de 60 minutos. Nesta condição foram obtidos um rendimento de $88,3 \%$ e 241 de grau de polimerização.
\end{abstract}

\section{INTRODUÇÃO}

Desde que foi introduzida no Brasil no século XVI, a cana-de-açúcar tem sido um dos pontos fortes da economia brasileira. Atualmente, o Brasil é o maior produtor de cana-deaçúcar no mundo, produzindo 633,26 milhões de toneladas na safra 2017/2018. Esta produção gerou uma quantidade estimada de 88,6 milhões de toneladas de bagaço (CONAB, 2018). Atualmente esse bagaço já não é mais considerado um resíduo, mas sim um coproduto devido a sua alta gama de utilidades, como por exemplo, etanol de $2^{\circ}$ geração, papel biodegradável e a celulose microcristalina, que é o foco deste trabalho. A celulose é um dos principais biopolímeros, tanto em quantidade quanto em utilidade. Entretanto, devido às propriedades de suas ligações químicas, não é possível a sua dissolução em água, limitando as áreas em que pode atuar.

Convencionalmente, a MCC é preparada ao tratar alfa-celulose com uma quantidade excessiva de um ácido mineral. As microfibras que compõem a alfa-celulose consistem em fases para-cristalinas e cristalinas. A porção para-cristalina é uma massa amorfa de cadeias de celulose, enquanto as regiões cristalinas compreendem feixes apertados de microcristais em um arranjo linear rígido (LEPPÄNEN et al., 2009).

A utilização da MCC pode se dar a partir da forma pura e seca de partículas finas em pó ou coprocessado com um polímero solúvel em água para fornecer uma forma coloidal. Assim, esta forma celulósica encontra aplicações nas indústrias farmacêuticas, cosméticas e de alimentos. Ainda na forma de pó, pode ser usada como aglutinante, comprimidos, além de agente de reforço na preparação de compósitos poliméricos diversos (TRACHE et al., 2016). 


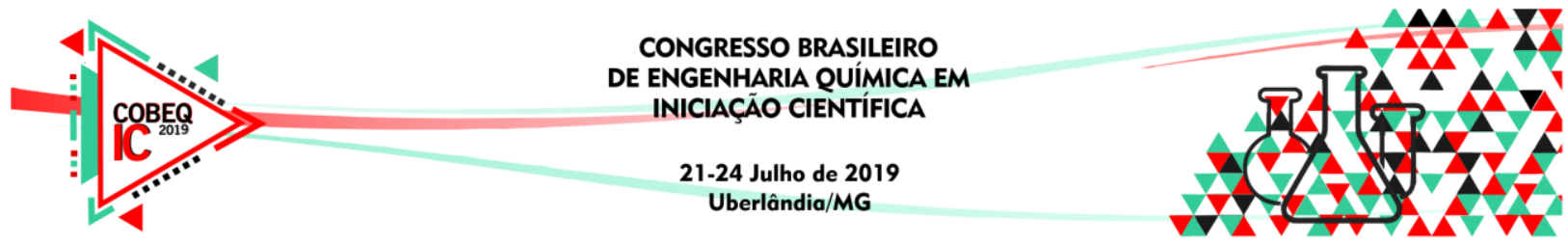

Devido às características como leveza, rigidez, natureza fibrosa, biodegradabilidade, atoxicidade, cristalinidade e renovabilidade, a MCC tornou-se atraente para diversos campos industriais, justificando um estudo mais aprofundado das condições de síntese deste material.

\section{MATERIAIS E MÉTODOS}

\subsection{Obtenção da Matéria-prima (Celulose Branqueada)}

Bagaço de cana-de-açúcar in natura doado pela Usina Virgulino de Oliveira, Unidade de Itapira, foi submetido a pré-hidrólises ácida e alcalina, além de uma sequência de branqueamento conforme descrito por Salim (2016).

Pré- hidrólise Ácida: Hidrólise ácida conduzida nas seguintes condições: tempo de reação de 30 minutos, temperatura de $121^{\circ} \mathrm{C}$ e razão sólido-líquido de $1: 10(\mathrm{~m} / \mathrm{v})$ e $100 \mathrm{mg}$ de ácido sulfúrico para $1 \mathrm{~g}$ de massa seca de bagaço (FONSECA, 2012).

Pré-hidrólise Alcalina: Hidrólise alcalina conduzida nas condições operacionais seguintes: tempo de reação de 60 minutos, temperatura de $98^{\circ} \mathrm{C}$ e razão sólido-líquido de 1:10 (m/v) e $100 \mathrm{mg}$ de $\mathrm{NaOH}$ para $1 \mathrm{~g}$ de massa seca de bagaço (FONSECA, 2012).

Sequência de branqueamento: $\mathrm{O}$ branqueamento foi realizado em seis etapas [sequência ECF (Element Chlorine Free)] nas condições otimizada. As etapas realizadas foram as seguintes: peróxido de hidrogênio $\left(\mathrm{H}_{2} \mathrm{O}_{2}\right)$, extração alcalina $(\mathrm{NaOH})$, hipocloração $(\mathrm{NaClO})$, extração alcalina, hipocloração e peróxido de hidrogênio (SALIM, 2016).

\subsection{Obtenção de Celulose Microcristalina}

Após as etapas de branqueamento, a celulose foi submetida a hidrólise principal com ácido sulfúrico sob refluxo. As condições experimentais estudadas seguiram um planejamento experimental do tipo fatorial fracionado $2^{(4-1)}$ com três repetições no ponto central. As variáveis controle investigadas e os respectivos níveis são apresentados na Tabela 1. Como variáveis resposta, foram escolhidos o grau de polimerização (GP) e o rendimento. O produto de cada ensaio foi lavado e secado em estufa a $60^{\circ} \mathrm{C}$ (EL-SAKHAWY; HASSAN, 2007).

Tabela 1 - Níveis e parâmetros utilizados para o estudo

\begin{tabular}{ccccc}
\hline \multirow{2}{*}{ NíVEIS } & \multicolumn{4}{c}{ VARIÁVEIS CONTROLE } \\
\cline { 2 - 5 } & Razão massa/solução ácida $(\mathrm{g} / \mathrm{ml})$ & $\mathrm{H}_{2} \mathrm{SO}_{4}(\mathrm{~mol} / \mathrm{L})$ & Temperatura $\left({ }^{\circ} \mathrm{C}\right)$ & Tempo $(\mathrm{min})$ \\
\hline-1 & $1: 15$ & 0,5 & 65 & 60 \\
0 & $1: 20$ & 1,0 & 75 & 90 \\
+1 & $1: 25$ & 1,5 & 85 & 120 \\
\hline
\end{tabular}

\subsection{Determinação do Grau de Polimerização (GP)}

O GP foi determinado de acordo com as Normas T 230 om-99 e NBR ISO 5351 (2012), que consiste na solubilização da MCC em solução de cuproetilenodiamina (CUEN) por 30 minutos. Esta solução é transferida para o viscosímetro do tipo Cannon-Fenske para determinação do tempo de escoamento $\left(t_{\text {sol }}\right)$. Antes disso foi medido o tempo de escoamento do solvente CUEN puro $\left(\mathrm{t}_{0}\right)$. Com esses valores se determina a viscosidade relativa e a 


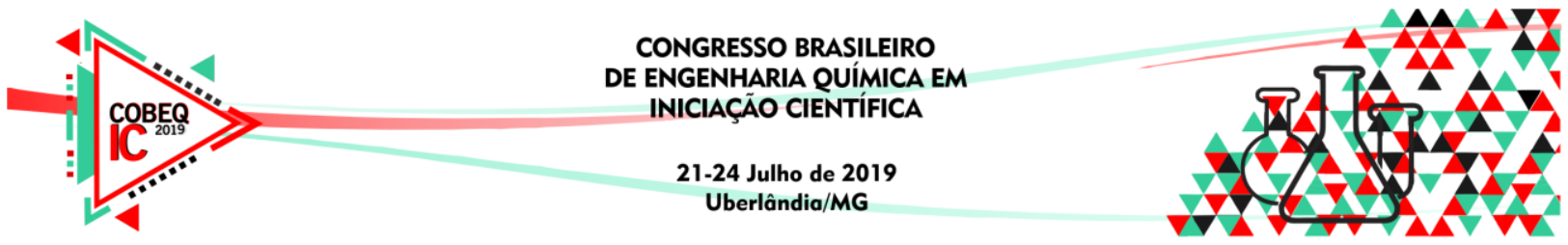

viscosidade intrínseca, a qual pode ser substituída na equação 1, determinando o GP, conforme proposto por Staudinger-Kuhn-Mark-Houwink (KLEMM, et. al., 1998).

$$
[\eta]=1,67 * \mathrm{GP}^{0,71}
$$

\subsection{Determinação do rendimento para a obtenção de MCC}

O rendimento da reação de obtenção de MCC [R (\%)] utilizando hidrólise ácida foi determinado pela divisão da massa seca final de MCC obtida $(\mathrm{m})$ pela massa de celulose inicial adicionada (M) como pode ser observado na Equação 2:

$$
R(\%)=(m / M) * 100
$$

\section{RESULTADOS E DISCUSSÕES}

Seguindo o planejamento experimental foram realizados os experimentos necessários a fim de obter os valores para as variáveis resposta, que podem ser observados na Tabela 2. Esses valores foram submetidos à análise estatística a um nível de significância de $95 \%$ com auxílio do software Minitab® 18, gerando os gráficos de Pareto e de efeitos individualizados (Figuras 1 e 2).

Tabela 2 - Matriz do planejamento fatorial $2^{(4-1)}$ com três repetições no ponto central com seus respectivos valores para as variáveis controle e resposta

\begin{tabular}{ccccc|cc}
\hline Ensaios & $\begin{array}{c}\text { Razão de massa/ } \\
\text { solução ácida }(\mathrm{g} / \mathrm{ml})\end{array}$ & $\begin{array}{c}\text { Concentração de } \\
\text { ácido }(\mathrm{mol} / \mathrm{L})\end{array}$ & $\begin{array}{c}\text { Temperatura } \\
\left({ }^{\circ} \mathrm{C}\right)\end{array}$ & $\begin{array}{c}\text { Tempo } \\
(\mathrm{min})\end{array}$ & Rendimento $(\%)$ & GP \\
\hline 1 & $1: 25$ & 0,5 & 85 & 60 & 85,6 & 335 \\
2 & $1: 25$ & 1,5 & 65 & 60 & 87,0 & 374 \\
3 & $1: 20$ & 1,0 & 75 & 90 & 96,4 & 348 \\
4 & $1: 15$ & 0,5 & 65 & 60 & 90,7 & 401 \\
5 & $1: 25$ & 1,5 & 85 & 120 & 79,6 & 223 \\
6 & $1: 15$ & 1,5 & 85 & 60 & 88,3 & 241 \\
7 & $1: 20$ & 1,0 & 75 & 90 & 96,4 & 319 \\
8 & $1: 15$ & 1,5 & 65 & 120 & 95,6 & 367 \\
9 & $1: 15$ & 0,5 & 85 & 120 & 92,2 & 306 \\
10 & $1: 20$ & 1,0 & 75 & 90 & 97,3 & 308 \\
11 & $1: 25$ & 0,5 & 65 & 120 & 92,1 & 391 \\
\hline
\end{tabular}

Ao analisar a Figura 1 nota-se que todos os fatores foram significativos para variável resposta rendimento. Entretanto, é perceptível que a razão massa/solução foi a de maior significância seguida pela temperatura, enquanto o tempo foi o fator de menor influência. A mesma análise pode ser feita observando os gráficos de efeitos principais individualizados, onde a variável controle razão/solução, apresentou maior inclinação entre os níveis avaliados.

A explicação para isso provavelmente deriva do aumento na interação da celulose com o ácido, intensificando a hidrólise mesmo com pequenos aumentos na concentração relativa do ácido, reduzindo a massa final do produto obtido. Logo, os fatores em seus níveis mais baixos tendem a aumentar o rendimento, pois a hidrólise ocorre com menor intensidade tanto nas regiões cristalinas quanto nas amorfas, gerando uma massa final maior do que em 


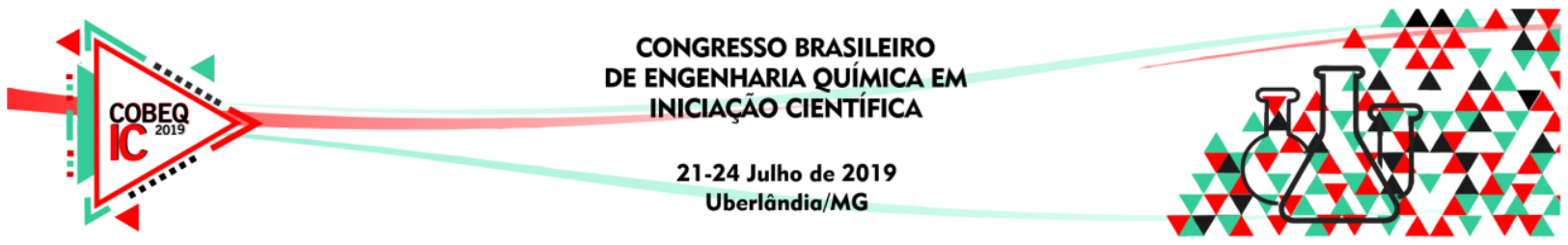

condições onde a hidrólise é intensificada. Por isso é importante ressaltar que um alto rendimento não necessariamente implica em uma qualidade alta, já que existe a possibilidade de haver uma fração amorfa considerável não hidrolisada em sua composição.

Quanto a variável reposta GP, e analisando o diagrama de Pareto, observa-se que apenas a temperatura apresentou significância estatística a um nível de confiança de 95\%, sendo que a concentração de ácido se apresentou bem próximo do limite de significância proposto. Estas afirmações podem ser corroboradas ao analisar os gráficos de efeitos principais individualizados, onde a influência da temperatura é bem intensa, em contrapartida a variável controle razão/solução apresentou pouca influência nos níveis estudados. Isto pode ser explicado considerando o fato de que teores maiores de ácido, combinados com temperaturas elevadas, intensificam o ataque ácido tanto na parte cristalina quanto amorfa da celulose, gerando cadeias moleculares menores com alta cristalinidade.

Figura 1 - Diagrama de Pareto e principais efeitos individualizados em função do rendimento através do planejamento $2^{(4-1)}$.
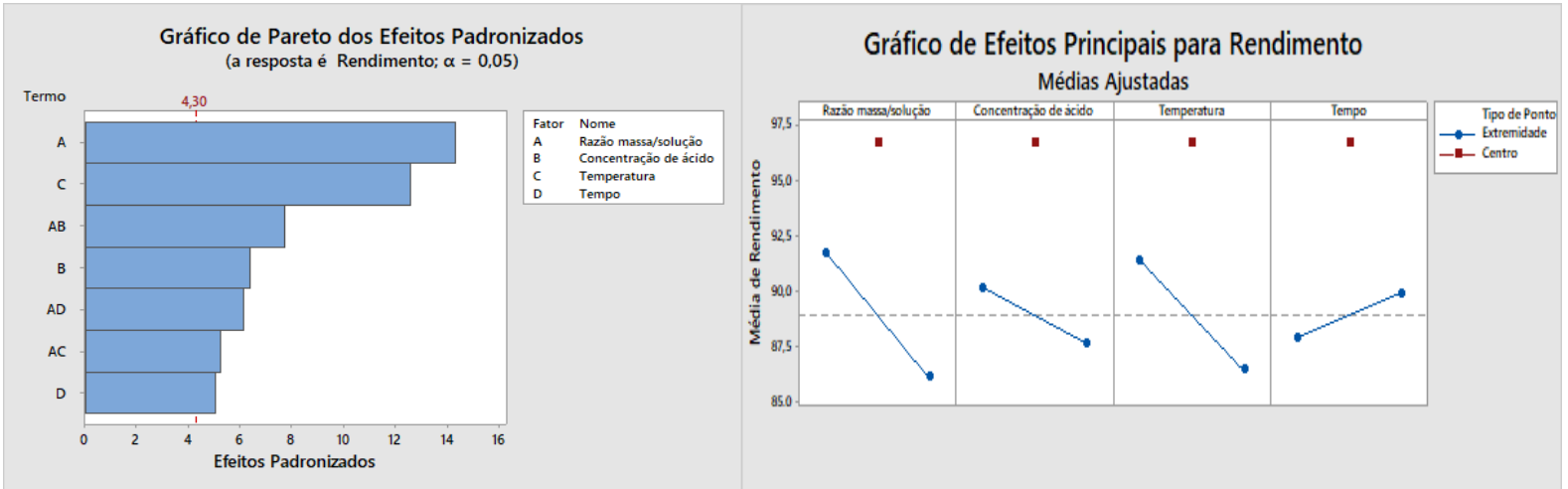

Figura 2 - Diagrama de Pareto e principais efeitos individualizados em função do grau de polimerização através do planejamento $2^{(4-1)}$.
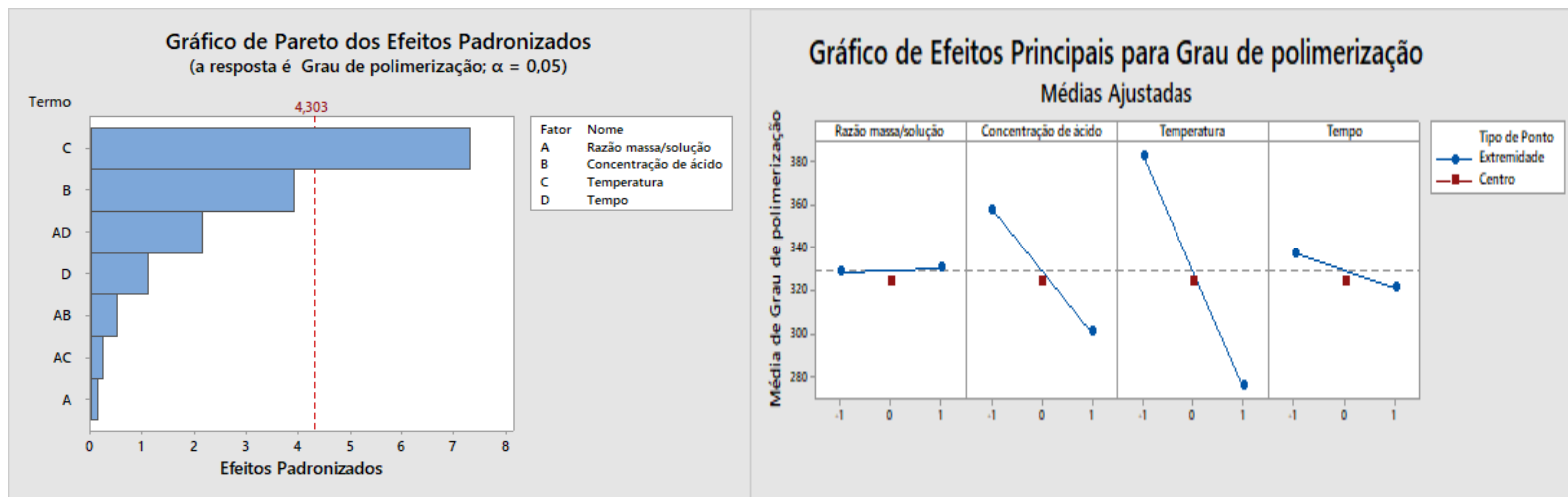

Ainda explorando os resultados estatísticos aplicando um teste de hipóteses (teste " $\mathrm{t}$ " de Student) foram gerados modelos lineares que visam representar os resultados obtidos em função das variáveis controle significativas (Equação 3 e 4).

$$
\begin{aligned}
& R(\%)=88,9-2,811 * R m s-1,252 * C_{A S}-2,473 * T+0,99 * t- \\
& 1,512 * \mathrm{Rms} * \mathrm{C}_{A S}-1,033 * \mathrm{Rms} * \mathrm{~T}-1,202 * \mathrm{Rms} * \mathrm{t}
\end{aligned}
$$




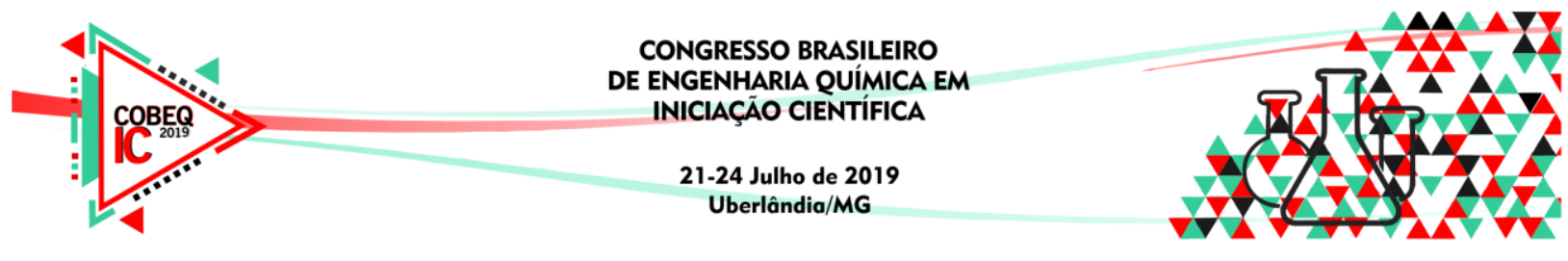

$$
\begin{aligned}
& G P=329,71+0,92 * R m s-1,252 * C_{A S}-53,42 * T-8 * t-1,81 * \mathrm{Rms} * \mathrm{~T}- \\
& 3,76 * R m s * C_{A S}+15,77 * \mathrm{Rms} * \mathrm{t}
\end{aligned}
$$

Sendo: $\mathrm{R}=$ rendimento; $\mathrm{Rms}=$ razão massa/solução ácida; $\mathrm{C}_{\mathrm{AS}}=$ conc. $\mathrm{H}_{2} \mathrm{SO}_{4} ; \mathrm{T}=$ temperatura e $\mathrm{t}=$ tempo.

Vale ressaltar, que no modelo apresentado acima, apenas a variável Temperatura apresentou significância estatística a 95\% de confiança.

A seguir foram realizadas as análises de variância para ambas as respostas, para confirmar se os modelos lineares e curvaturas apresentavam significância estatística poderiam apresentar curvatura significativa na região estudada (BARROS NETO; SCARMINIO; BRUNS, 2007). Esses resultados são apresentados nas Tabelas 3 e 4.

Tabela 3 - ANOVA para o rendimento pelo planejamento experimental $2^{(4-1)}$

\begin{tabular}{ccccc}
\hline Fonte de Variação & Graus de liberdade & SQ & QM & P-Valor \\
\hline Modelo Linear & 4 & 132,536 & 33,134 & 0,009 \\
Interação de $2^{\circ}$ Ordem & 3 & 38,372 & 12,791 & 0,024 \\
Curvatura & 1 & 133,181 & 133,181 & 0,002 \\
Erro & 2 & 0,617 & 0,309 & \\
Total & 10 & 304,707 & & \\
\hline
\end{tabular}

Tabela 4 - ANOVA para o grau de polimerização pelo planejamento experimental $2^{(4-1)}$

\begin{tabular}{ccccc}
\hline Fonte de Variação & Graus de liberdade & SQ & QM & P-Valor \\
\hline Modelo Linear & 4 & 29896,5 & 7474,1 & 0,055 \\
Interação de $2^{\circ}$ Ordem & 3 & 2129,2 & 709,7 & 0,398 \\
Curvatura & 1 & 49,9 & 49,9 & 0,765 \\
Erro & 2 & 855,9 & 428 & \\
Total & 10 & 32931,5 & & \\
\hline
\end{tabular}

Os resultados obtidos pela ANOVA para o rendimento mostram que tanto seu modelo linear como a interação são significativos (p-valores < 0,05). O teste de curvatura também se mostrou representativo, indicando que mesmo com um modelo linear ajustado, um modelo quadrático poderia ser indicado, com a vantagem de indicar regiões de máximo ou mínimo no intervalo avaliado. Por outro lado, para a variável resposta GP, a analise indica que o modelo linear representa melhor o conjunto de experimentos do que o modelo quadrático. A análise indica que o modelo linear representa melhor o experimento do que o modelo quadrático (pvalor $=0,055)$, pois a curvatura neste apresentou um $\mathrm{p}$-valor igual a 0,765 , muito superior ao valor limite de 0,05 .

De modo geral, entre os 11 experimentos realizados, o que apresentou os melhores resultados foi o ensaio 6, alcançando um rendimento de $88,3 \%$ e um baixo GP de 241 , nas condições reacionais de $85^{\circ} \mathrm{C}$ de temperatura, $1,5 \mathrm{~mol} / \mathrm{L}$ de $\mathrm{H}_{2} \mathrm{SO}_{4}$, tempo reacional de 60 minutos e uma razão massa/solução de 1:15 (g/mL). Estes resultados indicam que é possível obter um rendimento próximo de $90 \%$ e uma MCC de baixo GP, o que pode facilitar a aplicação deste material. 


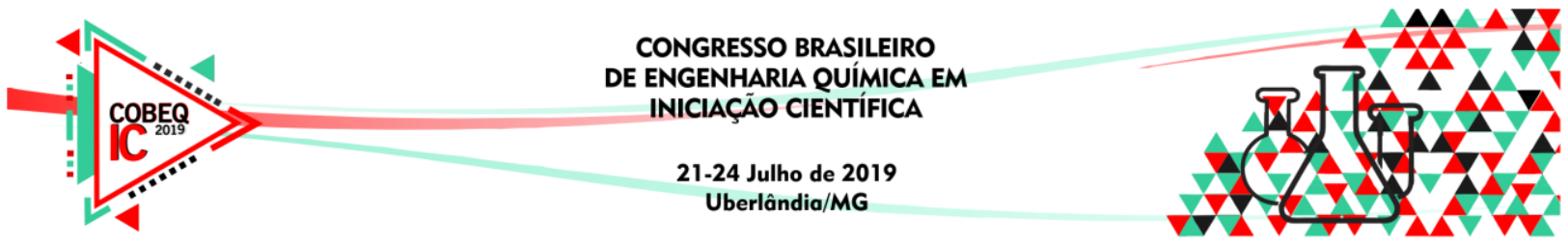

\section{CONCLUSÃO}

Através dos experimentos desenvolvidos foi possível verificar que a obtenção da MCC através da hidrólise ácida é um processo viável de obtenção deste produto celulósico. Contudo, visando o aperfeiçoamento das condições experimentais, estudos subsequentes podem ser realizados propondo um novo planejamento experimental que vise determinar um modelo quadrático mais representativo, através de um delineamento composto central rotacional (DCCR).

\section{REFERÊNCIAS}

BARROS NETO, B. de; SCARMINIO, I. S.; BRUNS, R. E. Planejamento e otimização de experimentos, $3^{\mathrm{a}}$ ed. Campinas: UNICAMP, 2007

Companhia Nacional de Abastecimento. Disponível em: <http://www.conab.gov.br/>. Acesso em 8 de abril de 2019.

EL-SAKHAWY, M.; HASSAN, M. L. Physical and mechanical properties of microcrystalline cellulose prepared from agricultural residues, Carb. Polym., V.67, 2007, Pg 1-10.

FONSECA, C. F. Uso combinado de processos visando a destoxificação de hidrolisado hemicelulósico. Dissertação (Mestrado) - Escola de Engenharia de Lorena, USP, 2012.

GALDÁMEZ, E. V. C. Aplicação das técnicas de planejamento e análise de experimentos na melhoria da qualidade de um processo de fabricação de produtos plásticos. Dissertação (Mestrado). Escola de Engenharia de São Carlos, USP, 2002.

KLEMM, D.; PHILIPP, B.; HEINZE, T.; HEINZE, U.; WAGENKNEEHT, W.; "Comprehensive Cellulose Chemistry", V.1 (1998).

LEPPÄNEN, K.; ANDERSSON, S.; TORKKELI, M.; KNAAPILA, M.; KOTELNIKOVA, N.; SERIMAA, R. Structure of cellulose and microcrystalline cellulose from various wood species, cotton and flax studied by X-ray scattering, Cellulose 16 (6) (2009).

SALIM C. S. Otimização do branqueamento do bagaço de cana para a obtenção de metilcelulose. Dissertação (Mestrado) - USP, Escola de Engenharia de Lorena, 2016.

TRACHE, D.; HUSSIN, M. H.; CHUIN, C. T. H.; SABAR, S.; FAZITA, M. R. N.; TAIWO, O. F. A.; HASSAN, T. M.; HAAFIZ, M. K. M. Microcrystalline cellulose: Isolation, characterization and bio-composites application-A review, Int. J. Biol. Macromol., V.93, Part A, 2016, Pg 789-804. 